\title{
Directed closure measures for networks with reciprocity
}

\author{
C. Seshadhri, ${ }^{*}$ Ali Pinar, ${ }^{\dagger}$ Nurcan Durak,${ }^{\ddagger}$ and Tamara G. Kolda ${ }^{\S}$ \\ Sandia National Laboratories, Livermore, CA, USA
}

(Dated: October 30, 2018)

\begin{abstract}
The study of triangles in graphs is a standard tool in network analysis, leading to measures such as the transitivity, i.e., the fraction of paths of length 2 that participate in triangles. Real-world networks are often directed, and it can be difficult to "measure" this network structure meaningfully. We propose a collection of directed closure values for measuring triangles in directed graphs in a way that is analogous to transitivity in an undirected graph. Our study of these values reveals much information about directed triadic closure. For instance, we immediately see that reciprocal edges have a high propensity to participate in triangles. We also observe striking similarities between the triadic closure patterns of different web and social networks. We perform mathematical and empirical analysis showing that directed configuration models that preserve reciprocity cannot capture the triadic closure patterns of real networks.
\end{abstract}

\section{INTRODUCTION}

The study of triangles is by now a classic tool in the analysis of large-scale networks. The focus on triangles has its roots in a variety of disciplines: in social sciences as a manifestation of theories of edge formation $[1,2]$, in physics as a local measure of clustering [3], in biology as motifs [4]. Capturing triangle structure in generative models is also of great interest $[5,6]$. We consider the problem of studying triangles in directed networks. Most social, communication, cyber, and web networks are directed networks. In directed networks, it has been observed that there is generally a significant percentage of reciprocal edges [7-14]. Newman et al. [7] show that the fraction of such edges in commonly studied graphs is quite high (refer also to Table III), and subsequent studies underlined the importance of such edges in network formation and information diffusion $[8,11,13]$. The set of wedges (Figure 1) and triangles (Figure 2) involving directed and reciprocal edges holds information about the underlying dynamics $[1,4,15-18]$. But it is challenging to use this information to compare different graphs. We treat a directed graph as having two different types of edges: directed and reciprocal. A reciprocal edge is technically a pair of directed edges, $\{(i, j),(j, i)\}$, that we treat as a single reciprocal edge. In our figures, reciprocal edges are depicted as double-headed arrows. Treating reciprocal edges explicitly has been done since the triad census work of Holland and Leinhardt [1], and more recently for trade network analyses $[9,18]$. Observe that reciprocal edges are essentially undirected. In this paper, the total number of edges refers to the sum of the number of directed edges plus the number of reciprocal edges. Following [7], we define reciprocity of a graph, $r$, as the ratio of the number of reciprocal edges to the total number of edges.

\footnotetext{
* scomand@sandia.gov

† apinar@sandia.gov

‡ nurcan.durak@gmail.com

$\S$ tgkolda@sandia.gov
}

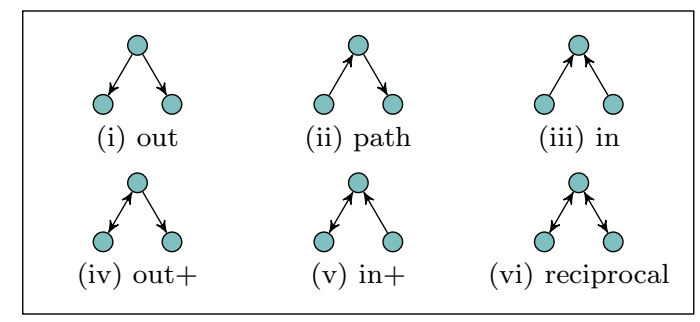

FIG. 1: Directed wedges

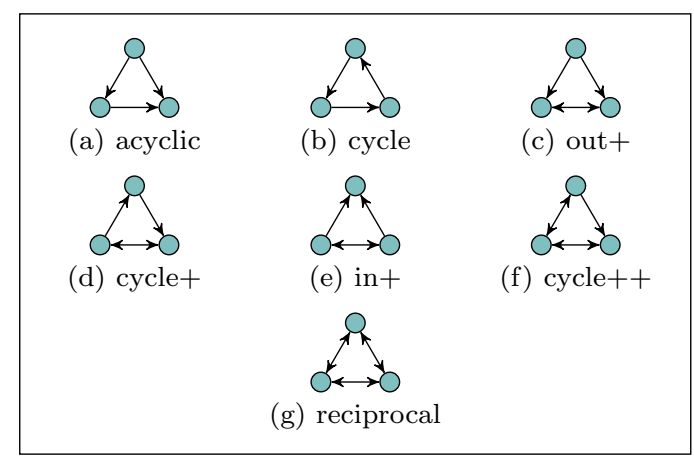

FIG. 2: Directed triangles

\section{A. Main results of this paper}

We generalize the classic notion of transitivity (pg. 243 of [19]), also called the global clustering coefficient, to directed graphs. We say a wedge is closed if it participates in a triangle. As described formally in §II, considering all possible directed wedge and triangle combinations yields a set of 15 directed closure values that provide a triadic summary of a directed graph. We perform experiments on a set of publicly available datasets and present the directed closure information in a succinct form that allows for a comparison of different graphs. This leads to a series of observations.

- Heterogeneity of closure: We find the closure fractions of wedges vary greatly depending on the wedge type. "In" wedges are typically the most numerous but 
are rarely closed. On the other hand, all other wedge types are much less numerous but are closed at a higher rate.

- Reciprocity inducing closure: For every graph we analyze, the presence of a reciprocal edge in a wedge greatly increases the probability of closure. In other words, wedges with reciprocal edges participate in triangles more frequently than wedges without any reciprocity.

- Transitivity correlates with reciprocity: Certain triangles are infrequent, specifically acyclic and cycle + triangles. The fact that cycles without reciprocal edges are so rare suggests that transitivity and reciprocity go hand in hand. This appears to validate the importance of transitivity, as posited by Holland and Leinhardt [1] in the social science community.

- Directed closures not explained by degree structure: It has been observed that many network patterns can be explained by the degree structure. Configuration models involving reciprocity were introduced by Garlaschelli and Loffredo [9] and also studied in [20]. These models have been observed to give better predictions of triadic structure $[18,21]$. However, we mathematically and empirically verify that these models do not explain the directed closure patterns of real networks.

\section{B. Previous work}

The earliest study of directed triads with reciprocity is in the social sciences, by Holland and Leinhardt [1]. They compute the triad census that counts the 16 different possible triads (including the 3 patterns with at most one edge). They also try to measure the effects of reciprocity in network formation. Skvoretz et al. [22, 23] use the triad census of predict various biases in network formation. In a more recent study, Faust [16] compares the structure of various graphs using the triad census. Most of this work has been restricted to small data sets having only a few hundred nodes. Counting triads has been referred to as motif finding in the bioinformatics community [4]. Directed triangle counts have been used to define enhanced modularity measures [24]. Simpler versions of triad census counts have also been used to analyze gaming data [17]. Szell et al. also perform triadic analysis on gaming data $[25,26]$. A classic local measure of triangle density is the clustering coefficient, introduced by Watts and Strogatz [3]. Fagiolo [15] proposes a local clustering coefficient measure for directed networks, though it ignores reciprocity. Ahnert and Fink [27] construct "clustering coefficients signatures" from these measures and classify directed networks. Recent work of Winkler and Reichardt [28] discusses the occurrence of the 16 different induced subgraphs on 3 vertices. They give an ingeneous model based on Steiner Triple Systems that can match more nuances of triad counts than other models. (But their model does not match the degree distributions.) A configuration model explicitly modeling reciprocity was given by Garlaschelli and Loffredo [9] and also studied in
[20]. There is further work showing its ability to match triadic patterns in trade networks $[18,21]$. Contrary to this work, our results show that for massive networks (like web and social networks), these models are unable to match triadic closure patterns. We have more discussion in $\S \mathrm{IV}$.

\section{DIRECTED CLOSURES VALUES}

We begin with some notation and introduction to the directed structures in Figure 1 and Figure 2. We stress that these types form a partition of all wedges and triangles. Since reciprocal edges are distinguished, we do not think of (say) the out+ wedge containing an out wedge. We use

$$
\begin{aligned}
& \psi \in\{\mathrm{i}, \mathrm{ii}, \ldots, \mathrm{vi}\}=\text { wedge type }, \text { and } \\
& \tau \in\{\mathrm{a}, \mathrm{b}, \ldots, \mathrm{g}\}=\text { triangle type. }
\end{aligned}
$$

Different triangle types naturally contain different types of wedges. This information is summarized by the function

$$
\chi(\psi, \tau)=\text { number of } \psi \text { wedges in a } \tau \text { triangle. }
$$

A table showing all values of $\chi(\psi, \tau)$ is provided in Table I. There are 15 nonzero entries in this table, and zeros are omitted for clarity. Given the degree of a node,

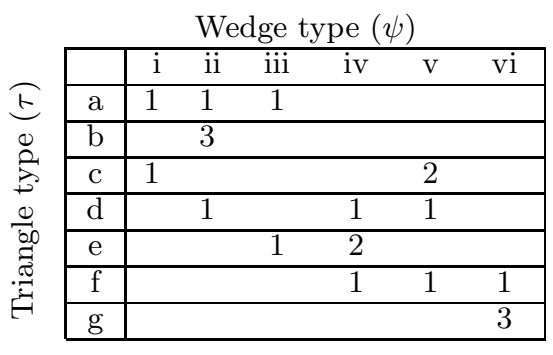

TABLE I: Wedges per triangle: $\chi(\psi, \tau)$.

we can calculate the number of each type of wedge it participates in. For vertex $v$, let

$$
W_{v, \psi}=\{\psi \text { wedges centered at node } v\}
$$

We compute $\left|W_{v, \psi}\right|$ given the degrees of $v$. Let

$$
\begin{aligned}
& d_{v}^{\leftarrow}=\text { indegree of } v, \\
& d_{v}^{\rightarrow}=\text { outdegree of } v, \text { and } \\
& d_{v}^{\leftrightarrow}=\text { reciprocal degree of } v .
\end{aligned}
$$

From these values, we can calculate $\left|W_{v, \psi}\right|$ for any vertex, as summarized in Table II. To define the directed closure values, we first define

$$
\begin{aligned}
W_{\psi} & =\bigcup_{v} W_{v, \psi}=\text { set of all wedges of type } \psi, \text { and } \\
T_{\tau} & =\text { set of all triangles of type } \tau .
\end{aligned}
$$




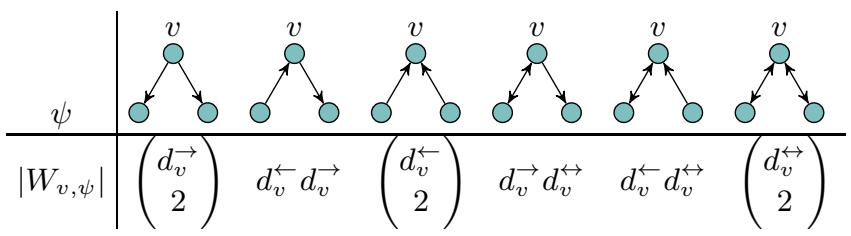

TABLE II: Number of $\psi$ wedges per vertex

We define the directed $(\psi, \tau)$-closure, $\kappa_{\psi, \tau}$, as the fraction of $\psi$-wedges that are $\tau$-closed, i.e.,

$$
\kappa_{\psi, \tau}=\frac{\chi(\psi, \tau)\left|T_{\tau}\right|}{\left|W_{\psi}\right|} .
$$

Note that if a type $\tau$ triangle contains no type $\psi$ wedge, then this quantity is zero because of $\chi(\psi, \tau)$. This definition is consistent with the undirected notion of transitivity. If we let $W$ and $T$ denote the set of all wedges and triangles, respectively, in an undirected graph, then the transitivity is defined as $\kappa=3|T| /|W|$. In this case, we know that three wedges participate in every triangle, which is the analogue for $\chi(\psi, \tau)$.

\section{OBSERVATIONS ON DIRECTED CLOSURE VALUES}

We analyze the directed closure properties of various real graphs, whose properties are presented in Table III. In this table, $r$ denotes the reciprocity and $\kappa$ denotes the undirected transitivity.

\section{A. Representations}

Figures 3-8 illustrate the $\kappa_{\psi, \tau}$ values. We explain using the example of web-Google [29] in Figure 3. The percentage of each triangle is shown at the top of each figure, along with the color code for the triangle, e.g., $10 \%$ of the triangles in web-Google are reciprocal, and these are represented by the color pink. The percentage of each wedge type is shown along the $x$-axis. For instance, $90 \%$ of the wedges in web-Google are in wedges. The height of the bar above each wedge denotes the rate at which that wedge is closed. For instance, $50 \%$ of the out wedges close for web-Google. The color codes on the bars show the type of triangle that the wedge becomes. For instance, the vast majority of closed out+ wedges in web-Google become in + triangles (per the green color). Finally, the transitivity $(\kappa)$ of the undirected graph is marked by a thick dashed line. For web-Google, $\kappa=0.055$.

\section{B. Similarities of directed closure rates}

Figures 3-5 show the closure charts for three different web graphs: web-Google, web-Stanford, and webBerkStan [29]. These graphs have vertices for web pages

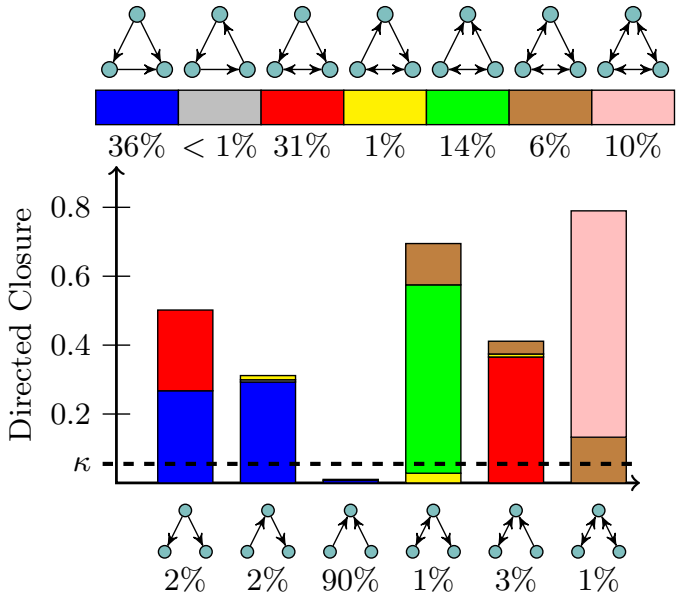

FIG. 3: Directed closure for web-Google

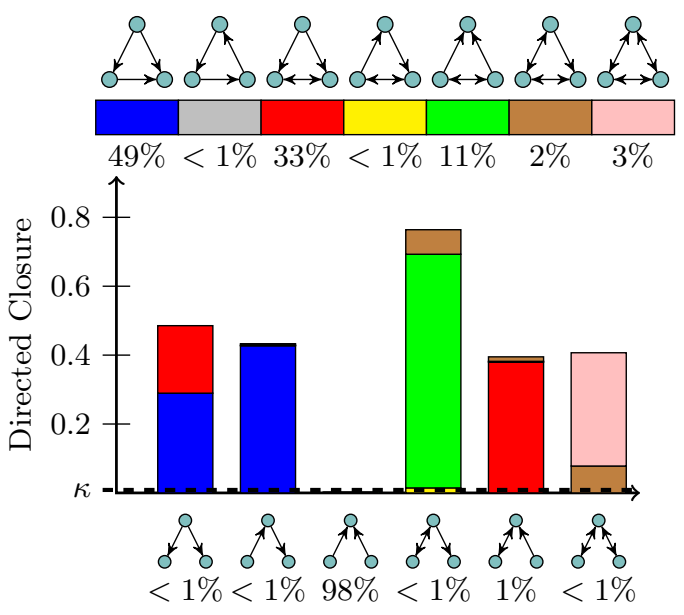

FIG. 4: Directed closure for web-Stanford

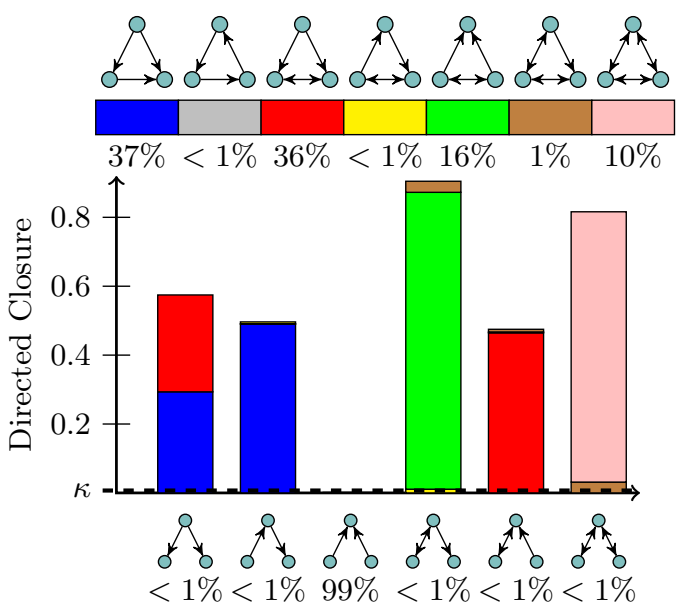

FIG. 5: Directed closure for web-BerkStan 


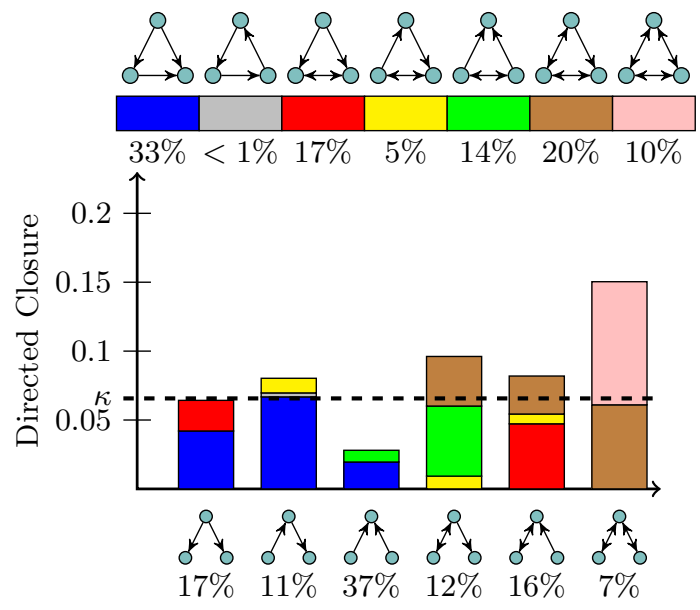

FIG. 6: Directed closure for soc-Epinions1

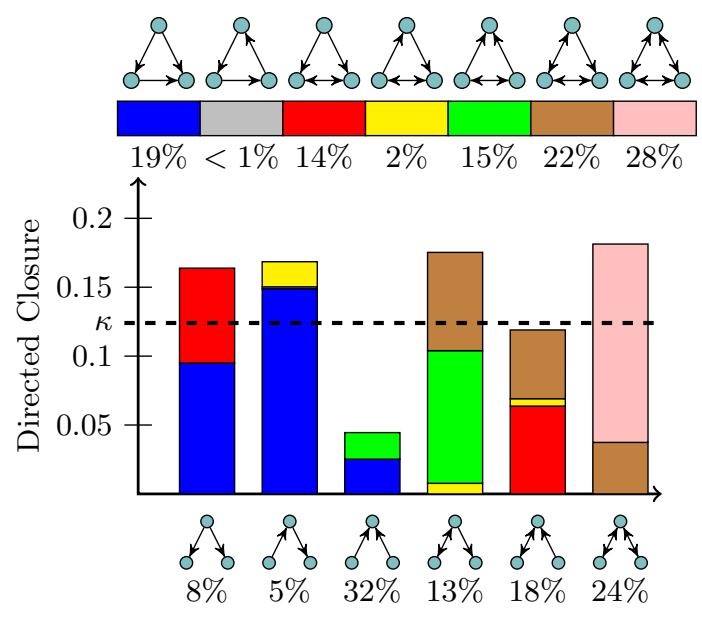

FIG. 7: Directed closure for livejournal

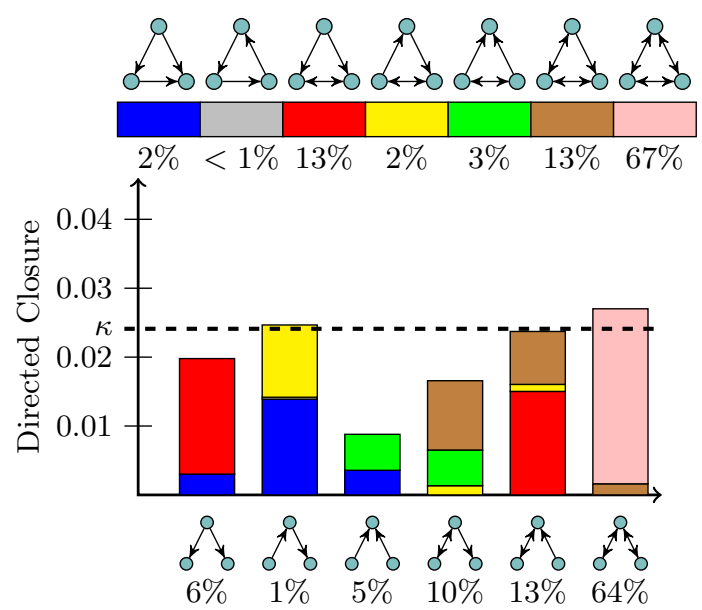

FIG. 8: Directed closure for soc-Slashdot0902
TABLE III: Properties of the graphs

\begin{tabular}{rrrcc} 
Graph Name & Vertices & Edges & $r$ & $\kappa$ \\
\hline amazon0505 & $410 \mathrm{~K}$ & $3357 \mathrm{~K}$ & 0.55 & 0.162 \\
soc-Slashdot0902 & $82 \mathrm{~K}$ & $870 \mathrm{~K}$ & 0.84 & 0.024 \\
web-Stanford & $282 \mathrm{~K}$ & $2312 \mathrm{~K}$ & 0.28 & 0.009 \\
web-BerkStan & $685 \mathrm{~K}$ & $7601 \mathrm{~K}$ & 0.25 & 0.007 \\
wiki-Talk & $2394 \mathrm{~K}$ & $5021 \mathrm{~K}$ & 0.14 & 0.002 \\
web-Google & $876 \mathrm{~K}$ & $5105 \mathrm{~K}$ & 0.31 & 0.055 \\
soc-Epinions1 & $76 \mathrm{~K}$ & $509 \mathrm{~K}$ & 0.41 & 0.066 \\
web-NotreDame & $326 \mathrm{~K}$ & $1470 \mathrm{~K}$ & 0.52 & 0.088 \\
youtube-links & $1158 \mathrm{~K}$ & $4945 \mathrm{~K}$ & 0.79 & 0.006 \\
flickr-links & $1861 \mathrm{~K}$ & $22614 \mathrm{~K}$ & 0.62 & 0.112 \\
soc-livejournal & $5284 \mathrm{~K}$ & $76938 \mathrm{~K}$ & 0.73 & 0.124 \\
\hline
\end{tabular}

and directed edges for web links. Figures 6-8 have the charts for three social networks [29]. The vertices of socEpinions correspond to the members of Epinions, a consumer review site. A directed edge between users shows a trust relationship originating from one user (these are signed by trust/distrust, which we ignore). The vertices of soc-Slashdot [29] are users and edges represent tagging as friend or foe. The vertices of soc-livejournal [30,31] are Slashdot users with edges denoting friendship (which is one-way). Observe the similarity of the closure rates and proportions of the different wedges for the web graphs, despite them being from different sources (and different sizes). The color patterns are remarkably similar, showing similar distributions of different closures. The social networks show more variation, but the overall structure of the charts is not far from the web graphs. In general, we note that in wedges rarely close and reciprocal and out + wedges generally close the most frequently.

\section{Heterogeneity of closure}

The heterogeneity of wedge closure is quite clear from all the closure charts. In the web graphs, the in wedges rarely close while all other wedges close at a rate of $25 \%$ or higher. On the other hand, the in wedges are the most frequent (90\% or more) so the undirected transitivity is always below 0.05 . The heterogenity is not as dramatic in the social networks, but there is some variation in closures over the wedge types. Consistently, in wedges close at the lowest rate and reciprocal wedges close most frequently.

\section{Effect of reciprocity on closure rates}

We can see from Figures 3-8 that wedges with reciprocal edges appear to close more frequently than those without. We do a comprehensive calculation on a variety of graphs in Figure 9 to show what proportion of wedges with $k \in\{0,1,2\}$ reciprocal edges closes into any triangle. Observe the strong influence of reciprocation in the closure rates. The average of chance of closure for a wedge without reciprocal edges is only $3 \%$, but this 
number goes to $23 \%$ if one of the edges is reciprocal and further increases to $38 \%$ when both edges are reciprocal. This finding is consistent with the earlier reports about reciprocal edges, indicating stronger ties between two vertices $[7,8,11,13]$. It also underscores how important it is to consider direction in networks since the rate of wedge closure depends on reciprocity of its edges.

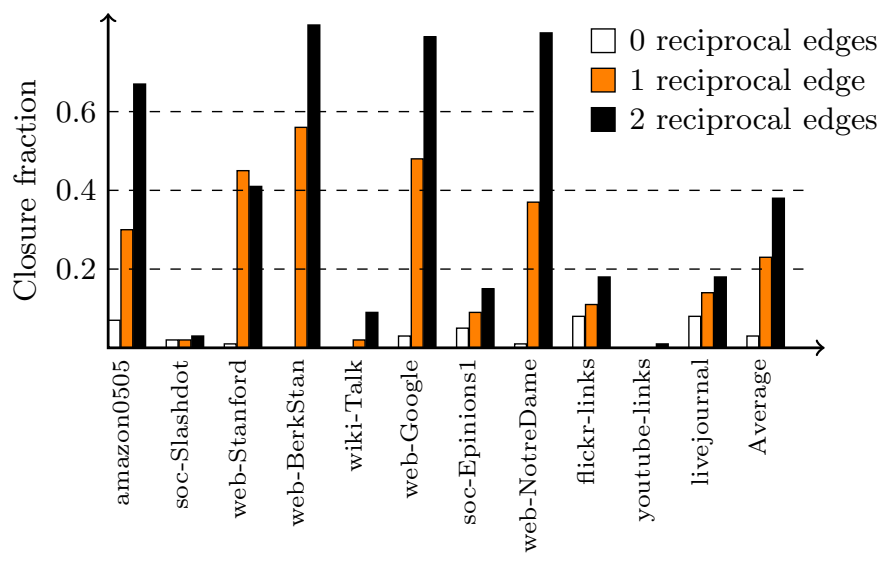

FIG. 9: Closure rates computed according to the number of reciprocal edges per wedge.

\section{E. The connection between reciprocity and cycles}

Throughout Figures 3-8, we notice the infrequency of cycle and cycle+ triangles (colored light blue and yellow, respectively), totaling less than $6 \%$ in all cases. These are two of the four triangles that contain a cycle, the other two being cycle ++ and reciprocal triangles (brown and pink, respectively). It is common to assume that a cycle indicates a strong tie between three vertices, and so we might hypothesize that reciprocation is expected. This is exactly what we see in Figure 10, where we illustrate the proportion of triangles that are cycles and the breakdown among the types. Almost all triangles with a cycle are either cycle ++ or reciprocal triangles. We almost never see any cycle triangles. Again, this is more evidence that reciprocal edges play an important role in graph structure. The results demonstrate the power of transitivity of real world networks: social relationships carried forward two steps (as a transitive relation) almost always lead to reciprocation.

\section{DIRECTED CLOSURES ARE NOT EXPLAINED BY DEGREES}

Could the different directed closures rates simply be a consequence of the (directed and reciprocal) degrees? We assert that this is not the case, by showing that a configuration model accounting for reciprocity cannot gen-

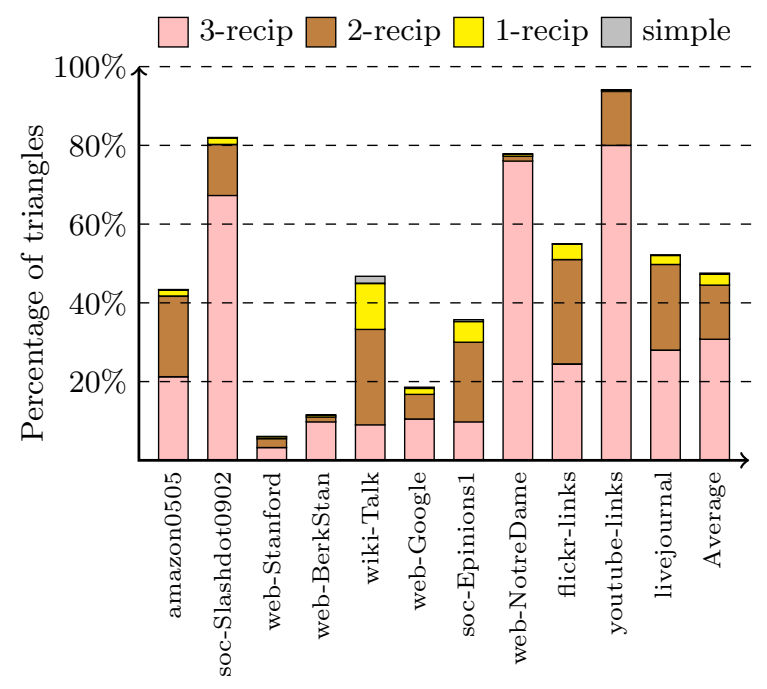

FIG. 10: Proportion of cycles in triangles, broken down by the number of reciprocal links.

erate the high $(\psi, \tau)$-closure values seen in the previous section. We explain the reciprocal configuration model of Garlaschelli and Loffredo [9] which respects both directed and reciprocal degrees. We assume that $d_{v}^{\leftarrow}, d_{v}$, and $d_{v}^{\leftrightarrow}$ are specified for every node. Let $m^{\leftarrow}=\sum_{v} d_{v}^{\leftarrow}$ or $m \rightarrow=\sum_{v} d_{v}$ denote the number of directed edges, and let $m^{\leftrightarrow}=\sum_{v} d_{v}^{\leftrightarrow}$ be twice the number of reciprocal edges. Conceptually, we assume that the model has an edge $i \rightarrow j$ with probability $d_{i} \rightarrow d_{j}^{\leftarrow} / m^{\rightarrow}$, and by a reciprocal edge between $i$ and $j$ with probability $d_{i}^{\leftrightarrow} d_{j}^{\leftrightarrow} / m^{\leftrightarrow}$. (We stress this is only an approximation, and there can be significant deviations from this approximation, as pointed out previously [32].) We give an approximation for expected $\kappa_{\psi, \tau}$, values. Previous analyses for transitivities on configuration graphs (see Newman [33], Park and Newman [32]) focus on undirected graphs, and it is not hard to generalize these calculations. Focus on the proportion of path wedges that become acyclic triangles, i.e., $\kappa_{i i, a}$, since it is consistently large for all networks we experiment on. Note that $\chi(i i, a)=1$, so $\kappa_{i i, a}=\left|T_{a}\right| /\left|W_{i i}\right|$. Observe that $\mathbb{E}\left[\left|W_{i i}\right|\right]=\sum_{v} d_{v}^{\leftarrow} d_{v}^{\rightarrow}$; furthermore, $\left|W_{i i}\right|$ is well-concentrated, so it is almost always close to its mean [34]. By linearity of expectation

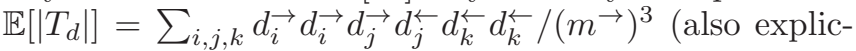
itly given by Squartini and Garlaschelli [18]). From this, we surmise

$$
\begin{aligned}
\mathbb{E}\left[\kappa_{i i, a}\right] & =\frac{\sum_{i, j, k} d_{i}^{\rightarrow} d_{i}^{\rightarrow} d_{j} d_{j}^{\leftarrow} d_{k}^{\leftarrow} d_{k}^{\leftarrow}}{\left(m^{\rightarrow}\right)^{3} \sum_{v} d_{v}^{\leftarrow} d_{v}} \\
& =\frac{\left(\sum_{j} d_{j} \overrightarrow{d_{j}} d^{\leftarrow}\right)\left(\sum_{i, k} d_{i} d_{i}^{\rightarrow} d_{k}^{\leftarrow} d_{k}^{\leftarrow}\right)}{\left(m^{\rightarrow}\right)^{3}\left(\sum_{v} d_{v}^{\leftarrow} d_{v}\right)} \\
& =\frac{\sum_{i} d_{i} \vec{i} d_{i} \sum_{k} d_{k}^{\leftarrow} d_{k}^{\leftarrow}}{\left(m^{\rightarrow}\right)^{3}} \\
& \approx \frac{4\left|W_{(i)}\right| \cdot\left|W_{(i i i)}\right|}{\left(m^{\rightarrow}\right)^{3}}
\end{aligned}
$$


We also look at the proportion of out+ wedges that become in triangles, i.e., $\mathbb{E}\left[\kappa_{i v, e}\right]$, another closure that is quite large. We have $\left|W_{i v}\right| \sum_{v} d_{v} d_{v}^{\leftrightarrow}$. We note that $2\left|T_{e}\right| \approx \sum_{i, j, k} d_{i} d_{i}^{\leftrightarrow} d_{j} \vec{j} d_{j}^{\leftrightarrow}\left(d_{k}^{\leftarrow}\right)^{2} /\left(m^{\rightarrow}\right)^{2} m^{\leftrightarrow}$. (We get the factor of two because type-e triangles have two typeiv wedges). Hence,

$$
\begin{aligned}
\mathbb{E}\left[\kappa_{i v, e}\right] & =\frac{\sum_{i, j, k} d_{i}^{\rightarrow} d_{i}^{\leftrightarrow} d_{j} d_{j}^{\leftrightarrow} d_{k}^{\leftarrow} d_{k}^{\leftarrow}}{\left(m^{\rightarrow}\right)^{2} m^{\leftrightarrow} \sum_{v} d_{v} d_{v}^{\leftrightarrow}} \\
& =\frac{\sum_{j} d_{j} d_{j}^{\leftrightarrow} \sum_{k} d_{k}^{\leftarrow} d_{k}^{\leftarrow}}{\left(m^{\rightarrow}\right)^{2} m^{\leftrightarrow}} \\
& \approx \frac{\left|W_{i v}\right| \cdot 2\left|W_{(i i i)}\right|}{\left(m^{\rightarrow}\right)^{2} m^{\leftrightarrow}} .
\end{aligned}
$$

Finally, consider reciprocal wedges that become reciprocal triangles, i.e., $\mathbb{E}\left[\kappa_{v i, g}\right]$, another closure that is often large. This is equivalent to an undirected calculation involving only reciprocal edges. Hence

$$
\mathbb{E}\left[\kappa_{v i, g}\right] \approx \frac{4\left|W_{v i}\right|^{2}}{\left(m^{\leftrightarrow}\right)^{3}} .
$$

In Figure 11, we show the ratio of the predicted closure values given by the equations above and the true closure values for the six networks we have been analyzing. Other than the graph soc-Slashdot0902, all other predictions are much smaller than the true value. For the web networks, the prediction is many order of magnitudes smaller than the true value. This is strong evidence that the directed closures cannot be explained by the degrees alone. To show the statistical significance of

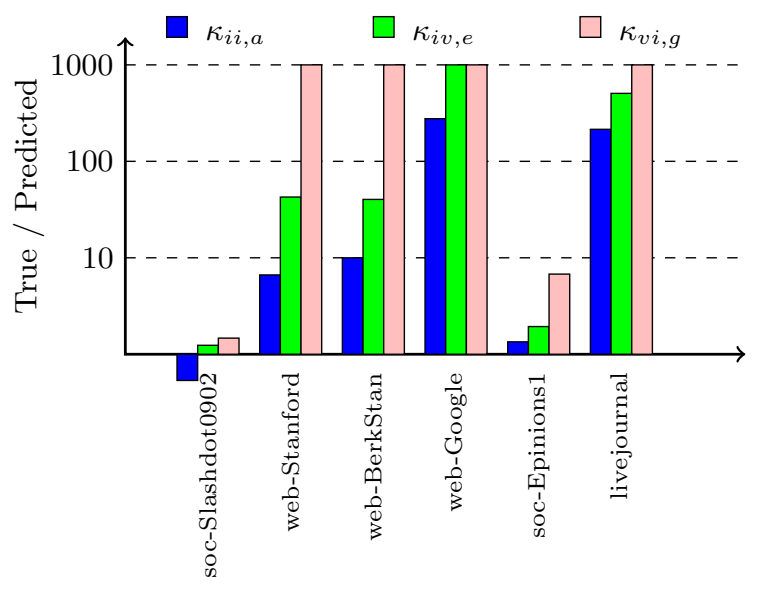

FIG. 11: True vs. predicted ratio for several directed closures. (For convenience, we truncate at a factor of 1000.)

these results, we compute the $Z$-scores for the results of the configuration model as compared to the true data. We observe the value of a directed closure value for multiple realizations of the configuration model. Let $X$ denotes that random variable with mean $\mu$ and variance $\sigma$. The Z-score is $(X-\mu) / \sigma$, i.e., the number of standard deviations the observation is from the mean of the models. We empirically estimate the mean and variance by generating 100 random instances from the configuration model. We present the results in Figure 12. Other than for $\kappa_{i i, a}$ in soc-Slashdot0902, all other $Z$-scores are positive. This is in excellent agreement with Figure 11 and our mathematical argument that the configuration model underestimates the directed closures (except in one case). Observe that the $Z$-scores are extremely large, which is an indication of statistical significance of the directed closure values for real networks.

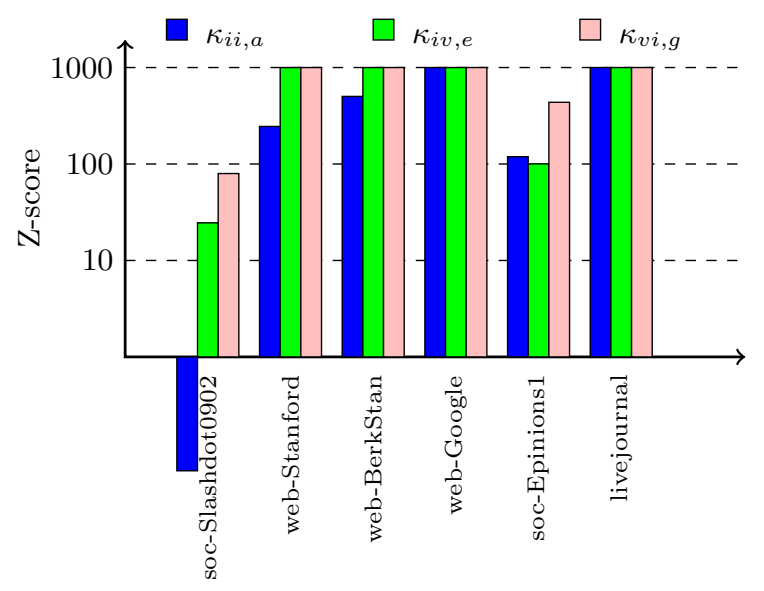

FIG. 12: Z-scores for several directed closures. (For convenience, we truncate all values at a factor of 1000.)

\section{CONCLUSIONS}

We perform a detailed study of directed triangles in massive networks, by defining the set of directed closure measures. These quantities reveal a surprising amount of information about directed graphs. We observe heterogeneity in closure rates of different wedges, the impact of reciprocity on closure rates, and the power of transitivity in the structure of triangles. We mathematically and empirically justify the statistical significance of these measures on real networks. We argue that is of great interest to design network models that recreate the directed closure patterns of real-world networks.

\section{ACKNOWLEDGMENTS}

This work was funded by the GRAPHS Program at DARPA, the applied mathematics program at the United States Department of Energy, and by an Early Career Award from the Laboratory Directed Research \& Development (LDRD) program at Sandia National Laboratories. Sandia National Laboratories is a multi-program laboratory managed and operated by Sandia Corporation, a wholly owned subsidiary of Lockheed Martin Corporation, for the U.S. Department of Energy's Na- 
[1] P. W. Holland and S. Leinhardt, American Journal of Sociology 76, 492 (1970).

[2] R. S. Burt, American Journal of Sociology 110, 349 (2004)

[3] D. Watts and S. Strogatz, Nature 393, 440 (1998).

[4] R. Milo, S. Shen-Orr, S. Itzkovitz, N. Kashtan, D. Chklovskii, and U. Alon, Science 298, 824.

[5] C. Seshadhri, T. G. Kolda, and A. Pinar, Physical Review E 85, 056109 (2012), 10.1103/PhysRevE.

[6] N. Durak, A. Pinar, T. G. Kolda, and C. Seshadhri, in ACM International Conference on Information and Knowledge Management (2012).

[7] M. E. J. Newman, S. Forrest, and J. Balthrop, Phys. Rev. E 66, 035101 (2002).

[8] D. Garlaschelli and M. I. Loffredo, Phys. Rev. Lett. 93, 268701 (2004).

[9] D. Garlaschelli and M. Loffredo, Physical Review Letters 73, 015101 (2006).

[10] G. Zamora-Lopez, V. Zlatic, C. Zhou, H. Stefancic, and J. Kurths, Physical Review E 77, 016106 (2008).

[11] A. Mislove, H. S. Koppula, K. P. Gummadi, P. Druschel, and B. Bhattacharjee, in WOSN'08 (ACM, 2008) pp. 25-30.

[12] V. Zlatic and H. Stefancic, Phys. Rev. E 80, 016117 (2009).

[13] H. Kwak, C. Lee, H. Park, and S. Moon, in $W W W$ '10 (ACM, 2010) pp. 591-600.

[14] T. Squartini, F. Picciolo, F. Ruzzenenti, and D. Garlaschelli, Scientific Reports 3 (2013).

[15] G. Fagiolo, Phys. Rev. E 76, 026107 (2007).

[16] K. Faust, Social Networks 32, 221 (2010).

[17] S. Son, A. R. Kang, H.-c. Kim, T. Kwon, J. Park, and H. K. Kim, PLoS ONE 7, e33918 (2012).
[18] T. Squartini and D. Garlaschelli, Lecture Notes in Computer Science 7166, 24 (2012).

[19] S. Wasserman and K. Faust, Social Network Analysis: Methods and Applications (Cambridge University Press, 1994).

[20] N. Durak, T. G. Kolda, A. Pinar, and C. Seshadhri, in Proc. IEEE 2nd Int. Workshop on Network Science 85.0561(20.13) also available as arxiv:1210.5288.

[21] G. Fagiolo, T. Squartini, and D. Garlaschelli, Journal of Economic Interaction and Coordination 8, 75 (2013).

[22] J. Skvoretz, Social Networks 12, 217 (1990).

[23] J. Skvoretz, T. J. Faroro, and F. Agneesens, Social Networks 26, 113 (2004).

[24] B. Serrour and A. A. an S. Gomez, Computer Communications 34, 629 (2011).

[25] M. Szell and S. Thurner, Social Networks 32, 313 (2010).

[26] M. Szell, R. Lambiotte, and S. Thurner, Proceedings of the National Academy of Sciences 107, 13636 (2010).

[27] S. E. Ahnert and T. M. A. Fink, Phys. Rev. E 78, 036112 (2008).

[28] M. Winkler and J. Reichardt, Phys. Rev. E 88, 022805 (2013)

[29] SNAP, "Stanford Network Analysis Project," Available at http://snap.stanford.edu/.

[30] F. Chierichetti, R. Kumar, S. Lattanzi, M. Mitzenmacher, A. Panconesi, and P. Raghavan, in KDD (2009) pp. 219-288.

[31] "Laboratory for web algorithmics," Available at http://law.di.unimi.it/webdata/ljournal-2008/.

[32] M. E. J. Newman and J. Park, Phys. Rev. E 68, 036122 (2003).

[33] M. E. J. Newman, SIAM Review 45, 167 (2003).

[34] T. Britton, M. Deijfen, and A. Martin-Löf, Journal of Statistical Physics 124 (2006). 